\title{
CONCEPCIONES DE CONFLICTO ESCOLAR: UN ESTUDIO DEL CONFLICTO DESDE LA PERSPECTIVA DE LOS ESTUDIANTES
}

Carlos Enrique Cabrera Arismendy ${ }^{1}$, Dina García Pérez ${ }^{2}$, Carlos Andrés Tabares Ramírez ${ }^{3}$

1. Universidad del Quindío Facultad de Educación, Programa de licenciatura en educación física y deportes.

2. Docente Educación Física, Recreación y Deportes IE María Auxiliadora (Santuario - Rda.).

3. Programa de licenciatura en educación física y deportes, Docente de Aula, Secretaria de Educacion de Armenia IE La Adiela.

\begin{abstract}
RESUMEN
El objetivo del artículo Concepciones de Conflicto Escolar: Un estudio del conflicto desde la perspectiva de los estudiantes, se centra en divulgar los hallazgos de la investigación homónima sobre las concepciones de conflicto que tienen los estudiantes de una Institución Educativa ubicada en Pereira, Risaralda, desde el enfoque de las teorías implícitas. Se plantearon como objetivos específicos identificar y analizar las concepciones de conflicto de los estudiantes del grupo focal elegido. La investigación fue cualitativa, de corte interpretativo; se utilizaron dos técnicas: la entrevista semiestructurada y el grupo focal. El análisis mostró las divergencias y convergencias significativas en los discursos de los estudiantes respecto del conflicto escolar, lo que propicia nuevas concepciones de este fenómeno en las instituciones educativas. Un hallazgo considerable es que los estudiantes son conscientes de que las situaciones no son naturalmente conflictivas; consideran que el conflicto se origina por actitudes agresivas o intolerantes de sus pares.
\end{abstract}

Palabras claves: Concepciones, Teorías Implícitas, Conflicto, Contexto escolar.

\begin{abstract}
The article "Beliefs towardSchool Conflict: A Study of the Conflict based on Students' perspectives" focuses on sharing the results of the research about students' beliefs of the conflict based on the Underlying Theories Approach. The students of the research belonged to an Educational Institution in Pereira-Risaralda. The research aimed at identifying and analysing the beliefs that the chosen student focus group had about Conflict. Then, the research was qualitative, following an interpretive approach. A semi structured interview and a focus group were used as data collection techniques. The analysis showed meaningful differences and similarities in the students' opinions about school conflict, what results in new understandings of this phenomenon at schools. It is worthy saying that a significant finding was related to the fact that students know that these situations are not naturally conflicting. On the contrary, students think that conflict arises when aggressive or intolerant attitudes from pairs happen.
\end{abstract}

Keywords: Conceptions, implicit theories, conflict, school context. 


\section{INTRODUCCIÓN}

"El cambio educativo requiere también promover un cambio en las concepciones de los agentes educativos sobre el aprendizaje y la enseñanza",

(Pozo et. al., 2006)

En el país se viven múltiples situaciones conflictivas a las cuales la escuela no permanece ajena. Al interior de la misma se viven cotidianamente situaciones inherentes a la interacción entre personas, que van desde un simple saludo descortés, hasta la agresión verbal o física entre estudiantes. El conflicto se presenta en la institución escolar y más precisamente, en las interacciones, como un fenómeno que hace parte de las mismas; esta connaturalidad del conflicto escolar y su profunda incidencia en los procesos educativos, lo han convertido en un objeto de estudio para destacados investigadores como Escudero y Moreno (1992), Zuleta (1994), Jarés (1997), Cascón (2000), Uprimny (2001), Galtung (2003), entre otros.

Para dar cuenta de las concepciones de conflicto escolar que tienen los estudiantes, se parte del supuesto de que ellas se construyen durante su vida, en la interacción con los demás y en las diversas experiencias vividas por cada uno de ellos. Se trata de su manera particular de entender las relaciones humanas y de relacionarse con el otro, en fin, del sentido que tiene para los jóvenes el conflicto. Se busca poner en evidencia lo que ellos piensan y saben acerca del conflicto, este conocimiento resulta supremamente interesante para los investigadores en su rol de profesores y para ampliar la reflexión acerca del contexto escolar y de los ambientes educativos; razón por la que la investigación se realiza en un entorno real.

Respecto de las concepciones en general, debe decirse que toman un carácter regulador que guía las acciones, ofreciéndoles un modelo de interpretación del mundo: para Driver (1982, p. 5), las concepciones "influyen en cómo los alumnos se enfrentan a los problemas, las variables que consideran significativas y los factores que observan y a los que les prestan atención"; Giordan y De Vecchi (1995, p.11) afirman que: "las concepciones han pasado de entenderse como una estructura intuitiva e imprecisa, a tomarse como un concepto didáctico, como teorías implícitas que el individuo puede ir modificando a medida que pasa el tiempo", gracias a la apropiación de nuevos conocimientos y a la vivencia de nuevas situaciones.
En lo que toca a la caracterización del conflicto en el contexto de la escuela se atiende, entre otros presupuestos, a los de Estévez (2006, p.125), que estudia la influencia del ambiente familiar "para dar cuenta de las variables individuales, familiares y escolares que originan los problemas de comportamiento agresivo/ violento, problemas de victimización por los iguales, y problemas de rechazo escolar por los compañeros"; al igual que a Jiménez, Lleras y Nieto(2010), que en su trabajo La paz nace en las aulas: evaluación del programa de reducción de la violencia, realizan una revisión del concepto "ciclo de violencia" propuesto por Chaux (2003), haciendo hincapié en el impacto que tiene para el niño y el joven crecer en un ambiente hostil durante la infancia, al momento de establecer interacciones en el ciclo escolar.

Desde esta perspectiva, las concepciones de conflicto escolar de los estudiantes constituyen un interesante campo de estudio abordado desde la pregunta ¿Cuáles son las concepciones de conflicto de los estudiantes de la Institución Educativa objeto de estudio de la ciudad de Pereira?

\section{Materiales y métodos}

Para responder la pregunta de investigación se formula un objetivo general: interpretar las concepciones de conflicto que tienen los estudiantes de la I.E. elegida para el análisis. Los objetivos específicos fueron: identificar las concepciones de conflicto de los estudiantes y analizar, desde la perspectiva de los estudiantes, sus concepciones de conflicto.

Estos dos objetivos se desarrollan a partir de cinco categorías iníciales que son: definición de conflicto, origen del conflicto, modo de enfrentarlo, modo de solucionarlo y participantes. Estas cinco categorías fueron observadas a partir de la aplicación de dos instrumentos: primero, la entrevista sobre concepciones de conflicto, validada a través de prueba de expertos y pilotaje, que fue triangulada con una situación problema acerca de situaciones específicas de conflicto; y segundo un grupo focal sobre conflicto, en el que se plantea a los estudiantes cinco preguntas relacionadas con cada una de las categorías iníciales.

En atención a lo dicho, la investigación se nutre fundamentalmente en los referentes teóricos de Marrero, Rodrigo y Rodríguez (1993) y De la Cruz, Mateos, Martín, Pérez, Pozoy Scheuer (2006), en lo que tie- 
ne que ver con el campo de las concepciones desde el enfoque de las teorías implícitas; mientras que en lo relacionado con campo del conflicto los referentes teóricos más destacados fueron: Escudero y Moreno (1992), Zuleta (1994), Jarés (1997), Cascón (2000), Uprimny (2001) y Galtung (2003), entre otros.

Debe anotarse que de las tesis de Jarés se adoptaron los tres enfoques desde los cuales se categorizan los conflictos en la investigación: el tecnocrático positivista (aquel que califica el conflicto como algo negativo, indeseable, disfuncional o patológico que debe evitarse y corregirse), el hermenéutico interpretativo (que rechaza la visión mecanicista del tecnocrático positivista, la ideología del control, concibe cada situación como propia e irrepetible condicionada por las interpretaciones particulares de cada miembro de la organización, por lo que omite las influencias y repercusiones grupales) y el crítico (que admite y favorece el afrontamiento de determinados conflictos desde una perspectiva democrática y no violenta, que suponga el cuestionamiento del propio funcionamiento de la institución escolar, y con ello, la correlación de fuerzas que en la misma existen); a juicio del grupo investigador este soporte teórico es el de mayor pertinencia para contrastar las cinco categorías iníciales planteadas para la construcción de los instrumentos y el análisis de la información.

En lo que tiene que ver con el aspecto metodológico, se desarrolla una investigación cualitativa de corte interpretativo, cuya unidad de análisis son las concepciones de conflicto de los estudiantes; la unidad de trabajo la constituyen ocho estudiantes, entre los doce y los quince años de edad, pertenecientes a los grados sexto, séptimo y octavo de una Institución Educativa de la ciudad de Pereira.

El procedimiento se realizó en tres fases: en primer lugar, la descripción, en la que se organizan, sintetizan y analizan los hallazgos desde los instrumentos y categorías iníciales; en segundo lugar la categorización, en la cual, a partir de las categorías emergentes, se evidencian las concepciones identificadas en cada uno de los instrumentos; y en tercer lugar, la interpretación, en la que se contrastan los hallazgos de cada instrumento, para darle sentido a los discursos de los estudiantes.

El análisis de la información se planteó en cinco apartados que dan cuenta de cada una de las cinco categorías iníciales: en el primero, se trabajaron las definiciones que los estudiantes dan al conflicto; en el segundo, se dio cuenta de los orígenes del conflicto; en el tercero se abordó las maneras como los estudiantes enfrentan los conflictos; en el cuarto se ahondó sobre las soluciones que los estudiantes dan a los conflictos escolares y el quinto y último apartado, abordó el tema de los participantes en los conflictos escolares; este procedimiento que a primera vista se presenta como una visión fragmentada del conflicto, se integra en el análisis para dar cuenta del fenómeno estudiado desde una perspectiva integral u holística.

Uno de los hallazgos más significativo lo constituye encontrar que las concepciones de conflicto por parte de los estudiantes, no corresponden de manera sesgada o esquemática a una sola de las perspectivas planteadas por Jarés (1997) y, por el contrario, atienden de manera alternada a una y otra de las tres perspectivas señaladas. En síntesis, plantea la necesidad de abordar el conflicto, por parte de todas las personas involucradas e interesadas en el fenómeno educativo, desde las perspectivas en que este se manifieste, para así conocerlo y aprovecharlo mejor en la construcción de otros escenarios educativos y de competencias ciudadanas, en los cuales las diferencias estén articuladas en la tolerancia.

\section{Discusión y Resultados}

Para una mejor comprensión de la discusión y los resultados se presentan de manera previa la unidad de trabajo que colaboró durante el desarrollo del proceso metodológico, así como una muestra de los instrumentos a partir de los cuales se clasificó la información obtenida, y ejemplos de cómo fue organizada la misma.

Tabla 1 Unidad de Trabajo

\begin{tabular}{lcccc}
\hline ESTUDIANTE & GENERO & GRADO & EDAD & ESTRATO \\
\hline Estudiante 1 & $\mathrm{F}$ & 8 & 15 & 6 \\
Estudiante 2 & $\mathrm{F}$ & 8 & 14 & 2 \\
Estudiante 3 & $\mathrm{F}$ & 7 & 12 & 3 \\
Estudiante 4 & $\mathrm{M}$ & 8 & 14 & 1 \\
Estudiante 5 & $\mathrm{M}$ & 7 & 14 & 3 \\
Estudiante 6 & $\mathrm{M}$ & 6 & 12 & 5 \\
Estudiante 7 & $\mathrm{F}$ & 6 & 12 & 5 \\
Estudiante 8 & $\mathrm{F}$ & 8 & 14 & 2 \\
\hline
\end{tabular}


Tabla 2 Ejemplo de instrumentos utilizados

\begin{tabular}{|c|c|c|}
\hline CATEGORÍA & $\begin{array}{c}\text { TÉCNICAS E } \\
\text { INSTRUMENTOS }\end{array}$ & PREGUNTAS \\
\hline $\begin{array}{l}\text { Definición de conflicto. } \\
\text { Se pretendió que los estudiantes } \\
\text { expresen,de manera desprevenida } \\
\text { y natural, lo que creen que es un } \\
\text { conflicto. }\end{array}$ & Entrevista & $\begin{array}{l}\text { Pregunta A: } \\
\text { - Para ti ¿qué es conflicto? } \\
\text { Pregunta D: } \\
\text { - ¿Podrías darme un sinónimo de conflicto? }\end{array}$ \\
\hline $\begin{array}{l}\text { Orienta a llevar su respuestas para } \\
\text { cada categoría: definición, como } \\
\text { se genera, como lo enfrenta, posi- } \\
\text { ble solución y quienes actúan. }\end{array}$ & Situación problema & $\begin{array}{l}\text { - Única pregunta: } \\
\text { - Describe una situación conflictiva en la que hayas par- } \\
\text { ticipado o presenciado en tu colegio en el último año. En } \\
\text { esta descripción debes tener en cuenta aspectos como: } \\
\text { posibles causas, consecuencias, participantes y solucio- } \\
\text { nes que se le dieron o pudieron dar en el momento que } \\
\text { sucedió. Propón dos alternativas de solución a esta situa- } \\
\text { ción conflictiva. }\end{array}$ \\
\hline Como lo enfrenta & Grupo focal & $\begin{array}{l}\text { Pregunta C: } \\
\text {-Si en el colegio te ves envuelto en una situación conflic- } \\
\text { tiva ¿Cómo actuarias? Explica tu respuesta. }\end{array}$ \\
\hline
\end{tabular}

Tabla 3 Organización de la información. Entrevista

\begin{tabular}{|c|c|c|c|}
\hline Definición & 1. ¿Qué es conflicto? A & 2.Sinónimos de conflicto D & SÍNTESIS \\
\hline E. 3 & $\begin{array}{l}\text { Para mi conflicto es un problema en- } \\
\text { tre personas }\end{array}$ & $\begin{array}{l}\text { Problema, tropel, desacuerdo, in- } \\
\text { conformidad. }\end{array}$ & $\begin{array}{l}\text { Problema, desacuerdo e } \\
\text { inconformidad. }\end{array}$ \\
\hline SIINTESIS & $\begin{array}{l}\text { Aparecen } 2 \text { o más personas. Visto } \\
\text { como agresión o como problema. } \\
\text { Cuando es agresión se entiende desde } \\
\text { tres ámbitos: físico, psicológico y ver- } \\
\text { bal... Problema }\end{array}$ & $\begin{array}{l}\text { Problema, agresión, pelea, des- } \\
\text { acuerdo e inconformidad. }\end{array}$ & \\
\hline
\end{tabular}

Tabla 4 Situación problema

\begin{tabular}{|c|c|c|c|c|c|c|c|c|c|}
\hline Categoría & S. 1 & S. 2 & S. 3 & S. 4 & S.5 & S.6 & S.7 & S.8 & Síntesis \\
\hline Definición & $\begin{array}{l}\text { Irrespeto, } \\
\text { grosería, } \\
\text { son de- } \\
\text { m a s i a d o } \\
\text { groseros e } \\
\text { irrespetuo- } \\
\text { sos tanto } \\
\text { verbalmen- } \\
\text { te como fí- } \\
\text { sicamente }\end{array}$ & $\begin{array}{l}\text { Proble- } \\
\text { mas con } \\
\text { mi salud. }\end{array}$ & $\begin{array}{l}\text { Proble- } \\
\text { ma. }\end{array}$ & $\begin{array}{l}\text { Situación } \\
\text { muy ma- } \\
\text { luca }\end{array}$ & $\begin{array}{l}\text { Insultar- } \\
\text { le a la } \\
\text { mama al } \\
\text { otro. }\end{array}$ & $\begin{array}{l}\text { conflic- } \\
\text { to fue de } \\
\text { droga - } \\
\text { dicción }\end{array}$ & $\begin{array}{l}\text { Irrespe- } \\
\text { tarse }\end{array}$ & $\begin{array}{l}\text { Insulto } \\
\text { e n t r e } \\
\text { compa- } \\
\text { ñeras }\end{array}$ & $\begin{array}{l}\text { Problema } \\
\text {. Agresión } \\
\text { física y ver- } \\
\text { bal. } \\
\text {. Situación } \\
\text { desagrada- } \\
\text { ble. } \\
\text {. Consumo } \\
\text { de droga. } \\
\text {. Insulto e } \\
\text { irrespeto }\end{array}$ \\
\hline
\end{tabular}

\section{Concepciones de conflicto}

Las concepciones de conflicto que a continuación se presentan, tienen como referentes las técnicas aplicadas $\mathrm{y}$, más concretamente, la información recogida a través de ellas. Esta información se analizó por parte del grupo de investigación, a la luz de los referentes teóricos y los resultados de las investigaciones cita- das como antecedentes; el abordaje de la información se realizó a partir de las categorías que previamente se establecieron, tratando de hallar en los resultados regularidades, divergencias o convergencias y las particularidades propias del contexto y la población específicos que se estudiaron, dando como resultado las siguientes concepciones organizadas por categoría. 


\section{Definición}

Esta categoría da cuenta de las concepciones que tienen los estudiantes frente a la definición de conflicto; en este sentido, resulta válido afirmar que para esta primera categoría, la definición es un elemento que se une con otros para conformar una totalidad o conjunto que puede asumirse como la verdadera concepción sobre conflicto.

Las respuestas de los ocho estudiantes, son breves y directas y afrontan la pregunta sin reflexiones adicionales; tienen la estructura de una argumentación pues plantean una opinión personal y formulan una tesis acerca de lo que es un conflicto, de hecho, tres de las ocho respuestas inician con la expresión: "para mí conflicto es".

Del análisis de estas respuestas, surgen tres concepciones por parte de los estudiantes para definir el conflicto como: "un problema de enfrentamiento y agresión", "problema de convivencia" y como "un problema de comunicación".

En primer lugar definen el conflicto como un problema de "enfrentamiento y agresión". En esta definición se detectaron dos generalidades: la primera de ellas apunta a una concepción de conflicto que asume el enfrentamiento entre partes e inicia por un desacuerdo entre personas o grupos; la segunda, asume la agresión no sólo desde lo físico, sino también desde lo verbal y psicológico, algo que resultó relevante para la investigación, ya que, como lo sugiere Cascón (2000), desde la racionalidad de los estudiantes este tipo de maltrato verbal o psicológico en ocasiones resulta imperceptible o mal interpretado por los adultos. Un ejemplo de ello es que el gesto que para un estudiante puede ser ofensivo y lesivo, para un adulto no pasa de ser una mueca entre estudiantes.

"Para mí, conflicto es cuando una persona no dialoga con otra y se agreden física, verbal y psicológicamente" E7.

"Conflicto son dos personas que no se respe tan y reaccionan mal, digamos pues que cuando es un conflicto verbalmente utilizan un vocabulario soez muy grosero o física mente tiran como a maltratarse" E1.

En segundo lugar, los estudiantes definieron el conflicto como "un problema de convivencia", considerando este problema como un fenómeno que emerge como producto de la simple interacción entre las personas y sus diferentes formas de actuar y concebir el mundo, es decir, reconocen el conflicto como algo inherente a las relaciones sociales y, tal como lo plantea Jarés (1997, p. 108): “ el conflicto es un tipo de situación en que las personas o grupos sociales, buscan o perciben metas opuestas, afirman valores antagónicos o tienen intereses divergentes".

"Para mí, conflicto es algo como personas que no saben convivir" E6.

En tercer y último lugar, los estudiantes asumieron el conflicto como "un problema de comunicación"; comunicación asumida como algo, de acuerdo con Valderrama (2001, p.77) que va más allá de lo verbal y de la transmisión de información, e incluye la actitud tomada por las personas frente a las diferencias propias de las interacciones con otros, en este caso, en el entorno escolar.

"Para mi conflicto es, cuando una persona no se comunica con otra" E7

"Conflicto es una discusión entre dos compa ñeros o puede ser entre varias personas, tam bién pueden ser compañeras gente de la insti tución" E4.

\section{Origen}

En la interpretación de las concepciones que tienen los estudiantes acerca de conflicto, resultó un paso determinante indagar la manera en la que ellos perciben la génesis del conflicto; si bien la gran mayoría de las respuestas apunta hacia una causa inmediata o causa detonante sin dar cuenta de las razones profundas que a nivel del imaginario colectivo estén generando los conflictos, este ejercicio permitió recoger una valiosa información acerca de aquellas causas cotidianas de conflicto que inciden en la vida escolar. Agrupadas en categorías brindan una idea global de la génesis del conflicto desde la racionalidad del estudiante y permitieron al grupo investigador tener una versión más integral y holística, mediante su clasificación en tres grupos: por intolerancia, diferencia e inconformidad y a partir de las interacciones sociales.

Para la primera concepción, el origen del conflicto como "intolerancia", los estudiantes dan cuenta de la intolerancia como una situación de irrespeto, ausencia 
de mesura en el trato brindado por el otro o hacia el otro, y como una falta de aceptación frente a la ofensa, es decir, la intolerancia como generadora de conflicto parte de la forma inteligente o racional, según lo dice Ordas (2008), como se reacciona frente a la ofensa y/o la falta de aceptación que se tiene por el otro.

"Por la intolerancia, por irrespeto hacía mi compañero o a cualquier persona, profeso res, mi mama, mis hermanas, he personas de la calle, bueno en fin a cualquier persona" E1. "Cuando se dicen algo, entonces el otro se pone bravo y puede haber una pelea" E2

Una segunda concepción que apareció en las respuestas de los estudiantes hace referencia al conflicto que emerge o tiene lugar a la luz de "las diferencias e inconformidades" que se presentan en los grupos sociales y, en este caso específico, en la escuela. En esta concepción en particular los estudiantes en consenso reconocieron la diferencia de pensamiento, de acción $\mathrm{y}$ de personalidad como un agente detonador de conflicto. Si se sigue lo propuesto por Mejía (2001) toman la no aceptación de la diferencia, como posición previa al conflicto y toman el enfrentamiento como resultado de dicha actitud frente a la diferencia. Dicho de otra forma, los estudiantes reconocen que todos somos diferentes, sin embargo, tienen claro que el origen del conflicto en su entorno escolar parte del rechazo y de la inconformidad que se tiene precisamente por esa diferencia propia de las relaciones sociales.

"Para mí, hay conflicto entre los seres huma nos, porque las personalidades son diferen tes" E1.

"Por ejemplo alguno no le cayó bien al otro, entonces ya se van a un conflicto, porque hay muchas diferencias" E8.

Por último la tercera concepción acerca del origen de conflicto es tomada por los estudiantes como un problema de "interacciones sociales", es decir, el conflicto en la escuela se origina no sólopor la intolerancia y la interacción con el otro, diferente de mí, como ya lo mencionaba Martínez (2005, p.4), sino también por la interacción que se tiene con los elementos sociales propios del entorno escolar como: manuales de convivencia, estructuras físicas, horarios, rutinas, PEI, etc.; que entran en juego para chocar o transformar esas concepciones que cada estudiante trae, las cuales fueron construidas a través de su vida y la interacción en contextos diferentes a la escuela.

"Bullying, acoso o burla hacia un compañe ro, las roscas que se dan entre compañeros". E3

\section{Estrategias}

Esta categoría pretende dar cuenta de la manera personal en la que los estudiantes se ven afectados por el conflicto, desde sus sentimientos y emociones, cuando participan de manera directa y activa en un conflicto escolar y la manera en la que se ven afectados cuando son espectadores pasivos de un conflicto ocurrido en el escenario escolar. Frente a ello surgieron dos concepciones: Protagonista activo y Espectador. Respecto de la primera concepción, "Protagonista activo", aparecen en los estudiantes sentimientos y emoción escomo: resentimiento, rabia, satisfacción, poder, impotencia y la necesidad de resolver la situación lo más pronto posible, siendo esta una reacción personal en una situación de carácter intersubjetivo frente al conflicto. Dicho de otra manera, dependiendo del rol que ocupe el estudiante en el conflicto así mismo serán sus sentimientos y emociones. Si la posición es de agresor, tendrá sentimientos de satisfacción, poder, placer, teniendo en cuenta que está ejerciendo la acción dominante en el conflicto; mientras que quien está en la posición del agredido o el débil, manifestaría rabia, impotencia, miedo y la necesidad de que esa situación acabe. En conclusión, de acuerdo con lo expresado por Hoyos (1995), los sentimientos y emociones que emergen de esta concepción dependen directamente del rol que ocupe cada protagonista del conflicto.

"Bueno, eh eh, la verdad, pues me da mucha rabia, y si, resentimiento y mucha impoten cia (...)" E1.

"Siento rabia (...) siento la necesidad de re solverlo lo más pronto posible" E3.

En contraste y como segunda concepción, “el espectador", en este caso en particular el estudiante que hace las veces de espectador, al verse afectado manifiesta su sentimiento de indignación frente a la situación que está presenciando de manera adicional a los sentimientos y emociones que pueden surgir, si uno o varios de los actores del conflicto tiene alguna afinidad con él. Frente a lo anterior los estudiantes plantean que de haber afinidad, ellos harían todo por 
separarlos o, de ser necesario, accederían a ser protagonistas del conflicto o buscarían de inmediato la ayuda de un mediador; por otro lado, en el caso de no haber afinidad, los estudiantes manifiestan indignación frente al hecho y la necesidad de buscar (al igual que en el primer caso), un tercero que haga las veces de mediador, quien aportaría de forma potencial a la solución del mismo.

"Yo de una fueran o no mis amigos, llamaría a un profesor. Porque yo, no sabría qué ha cer" E8.

"Mi reacción depende de varias cosas, si es mi amigo, si yo siento algo por él, me daría mucha rabia y hasta accedería a la pelea (...) y bueno si yo no siento nada por él, si hiciera algo, seria sepáralos y llamar a alguien ma yor" E7.

\section{Soluciones}

Esta categoría da cuenta de la manera como los estudiantes creen que pueden dar solución a los conflictos, a partir de una única alternativa ya tocada por Jarés (1997) "el dialogo". Sin embargo, a pesar de este consenso, surge una contradicción: pese a que se reconoce el diálogo como el mejor mecanismo para la solución de conflictos, se evidencia la incapacidad de anteponer el diálogo frente a la reacción agresiva, al manifestar, nuevamente, la necesidad de intervención por parte de un tercero, adulto, conciliador, con capacidades reconocidas en el contexto para el manejo de conflictos.

"Dialogando, pero si las dos personas están alteradas, pues ahí sí, esperar, no sé, que pue da pasar, pero yo digo que ya después de pa san las cosas, hablar para que esto no vuelva a suceder" E1.

"Sí, claro con los profesores (...) dialogando con los estudiantes y les hacen entender mu chas cosas" E2.

"Ponerse los zapatos del otro, mirar cómo se puede estar sintiendo el otro y de acuer do a la situación buscar soluciones, creo que hablar con los superiores informarlos de lo que está pasando" E3.

En conclusión, para los estudiantes hay una distancia entre la concepción del diálogo como alternativa única de solución y la capacidad de llevar de forma autónoma esta concepción frente a una situación conflictiva real, demostrando de forma implícita la heteronomía que se tiene, frente al manejo del conflicto en la escuela.

\section{Actores}

La intención en esta categoría fue indagar por los roles y las posiciones que los estudiantes adoptan en el desarrollo del conflicto. Los roles que las personas asumen pueden cambiarse en el escenario del conflicto, de tal manera que resulta muy interesante conocer la mirada de los estudiantes que participaron en la investigación, respecto de la performance que ellos y las personas con quienes interactúan en el espacio escolar asumen cuando participan en un conflicto. De allí surgieron tres concepciones frente a los actores del conflicto: dos o más personas, agresor y agredido, y un mediador.

Frente a la primera concepción, los estudiantes reconocen que en su entorno social escolar, los conflictos se generan en la interacción con el otro y las diferencias propias de los agentes sociales, identificando necesariamente la presencia de dos o más personas para que se desarrollen situaciones conflictivas como ya lo proponía Martínez (2005).

"Entre varias personas, entre dos o más, o en tre grupos contra una persona" E6.

"El que inicia el problema, malgeniado, gro sero, malgeniado, y otro agredido o más tran quilo" E8.

Además esta concepción, en contraste con la diferencia como detonante del conflicto, reitera el reconocimiento de la misma como factor social, como ítem para la conformación de grupos, la categorización de rechazos o exaltación de acciones socialmente aceptadas, factores que implícitamente surgen o hacen mella frente a la reacción positiva o negativa que tomen los estudiantes frente al otro y esa diferencia producto de una construcción personal, que se ven enfrentadas en el contexto social escolar.

Como un segundo factor tenemos la concepción del rol "agresor y agredido", que destacade forma directa el perfil que para ellos deben tener los actores del conflicto, y que además originan como alternativas de acción las siguientes: primero, dos personas de naturaleza agresiva y segundo, una persona de naturale- 
za agresiva y un agredido, de actitud pasiva, sumisa y tranquila. De esta forma los estudiantes muestran desde su experiencia escolar qué personajes habitualmente son los que generan, participan, callan, evaden, etc. las situaciones conflictivas a las cuales se ven enfrentados en su quehacer como estudiantes.

"Casi siempre son peleas, uno fuerte y uno débil" E4.

"Casi siempre, vea, es el grosero, contra el más calmado o el más serio, o a veces son los dos groseros peleando" E2.

En tercer lugar vuelve aparecer la figura de "mediador" como en la categoría tres y cuatro, reafirmándose como un hallazgo relevante la importancia de un tercero, intermediario y mediador, como agente idóneo para la resolución del conflicto. Adicionalmente, los estudiantes le aportan unas características especiales a este mediador: primero, debe ser adulto; segundo, estar revestido de una autoridad para el manejo de estas situaciones; tercero, debe generar confianza y respeto; y cuarto debe intervenir entre los protagonistas del conflicto y generar pautas de manejo a nivel familiar.

"A mi mejor amiga, podría actuar como con sejera, es decir que sabe arreglar las cosas, si muy inteligente" E2.

"A los estudiantes de mi salón, porque para este ejercicio son los más recomendados, pues hay unos que tienen su forma buena" E4.

Finalmente, el recorrido descriptivo sobre las concepciones de conflicto escolar de los estudiantes, realizado a través de cada una de las cinco categorías iníciales establecidas, permitió al grupo investigador aproximarse a un fenómeno de presencia indiscutible en las interacciones humanas vividas en la escuela. El conflicto se develó como un elemento que cohesiona dichas interacciones, una especie de común denominador que varía en la forma en que se presenta, pero tiene una esencia común en la cognición compartida y en la cognición individual de los miembros de la comunidad educativa.

\section{Conclusiones}

Después de consolidar los datos de la investigación se encuentran coincidencias considerables con las propuestas de Estévez (2006) -en lo que se refiere a la influencia del entorno familiar y social en el desempeño y actitudes tomadas por el estudiante en el entorno escolar -, al igual que con lo planteado por Sánchez (2005) - respecto de que las actitudes agresivas de los estudiantes generan a su vez un comportamiento reflejo en quien se siente agredido o amenazado -.

Teniendo en cuenta los resultados obtenidos, en futuras investigaciones resultaría pertinente indagar acerca de si el conflicto genera un impacto psicológico negativo únicamente en quien es agredido, como parece ser a simple vista o, si por el contrario, también promueve efectos de este tipo en el agresor. Resultaría importante además indagar si el papel que representan en el entorno escolar los tres actores definidos del conflicto por parte de los estudiantes (Agresor, agredido y conciliador) perviven en su entorno familiar y social.

Otros puntos de partida para trabajos posteriores podrían enfocarse en las posibles nociones que tienen los docentes y padres de familia sobre el conflicto, lo cual redundaría en un panorama más amplio y completo de esta temática, al contrastarse con los resultados obtenidos en esta investigación. Igualmente, sería interesante realizar un tipo de análisis similar con grupos de estudiantes que pertenezcan a Instituciones Educativas de características económicas y sociales diversas, puesto que en varias ocasiones se ha hecho explícito que las divergencias de este tipo entre grupos de estudiantes, aunadas a un uso inadecuado de las redes sociales, promueve el enfrentamiento entre grupos de jóvenes provenientes de distintos colegios.

De lo evidenciado mediante las entrevistas se vuelve ostensible la necesidad de que la escuela lleve a cabo un proceso de transformación en cuanto a su concepción de conflicto, actitud frente al mismo y estrategias de resolución efectivas en las que puedan participar todos los actores que conforman la comunidad escolar. La escuela, tal y como se presenta hoy, es un colectivo que aún debe desarrollarse en el plano moral y comunicativo para poder visualizar en el conflicto algo positivo.

Para conseguir esa transición axiológica en la visión del conflicto las Instituciones Educativas deben promover la creación de espacios académicos transversales en los que se estimule el ejercicio dialógico, el desarrollo de las funciones argumentativas y propositivas del lenguaje, y la participación de todos los 
estudiantes y demás miembros de la comunidad, en situaciones que aborden de manera abierta la diversidad y la otredad, para que a partir de la misma se posibilite la socialización de las distintas posiciones en cuanto al conflicto, las posibilidades que brinda y el planteamiento de salidas conjuntas, conciliadas, cuando el mismo se presente.

\section{BIBLIOGRAFÍA}

- Cascón, S. (2000).Educar en y para el Conflicto. Barcelona: Universidad Autónoma de Barcelona.

- Chaux, E. (2003).“Agresión reactiva, agresión proactiva y el ciclo de la violencia”. Estudios Sociales. 1 (15), 47-58.

- de la Cruz, M., Martín, E., Mateos, M. de M., Pérez, M. del P., Pozo, J. I. y Scheuer, N. (2006).Nuevas formas de pensar la enseñanza y el aprendizaje. Las concepciones de profesores y alumnos. Barcelona: Graó

- De Vecchi, G. y Giordan, A. (1995). Los orígenes del saber. De las concepciones personales a los conceptos científicos. Sevilla: Diada.

- Driver, R. (1982).“Enseñanza de las ciencias”. Revista de investigación y experiencias didácticas. 4 (1), 3-15.

- Escudero, J. y Moreno, J. (1992).El Asesoramiento al Centro Escolar. Madrid: Comunidad Autónoma de Madrid.

- Estévez, E. (2006). "Relación entre el clima familiar y el clima escolar: el rol de la empatía, la actitud hacia la autoridad y la conducta violenta en la adolescencia". Revista Mexicana de Psicología. 1 (25), 119-128.

- Galtung, J. (2003).“Teoría del conflicto”. Revista Paz y conflictos. 1(2), 60-81.

- Hoyos, V. G. (1995).“Ética Comunicativa y Educación para la Democracia”. Revista iberoamericana de educación. 1(7). 65-91.

- Jarés, X. (1997).“El lugar del conflicto en la organización escolar, micropolítica en la escuela”. Revista iberoamericana de educación. 1 (15), 53-73.

- Jiménez, M., LLeras, J., Nieto, A. M. (2010).“La paz nace en las aulas: evaluación del programa de reducción de la violencia”.Colombia Educación y Educadores.13 (3), 347-359.

- Martínez Alier, J. (2005).El Ecologismo de los Pobres. Conflictos Ambientales y Lenguajes de Valoración. Barcelona: Icaria.

- Mejía, M. (2001).“Construir educativamente el conflicto. Hacia una pedagogía de la negociación cultural”. Nómadas. 1 (15), 24-39.

- Ordas, N. (2008).Conflicto y violencia cultural en Colombia: propuesta de transformación frente a la escuela. Medellín: Corporación Correcaminos, Give To ColombiayTheMcMillan Children's Foundation.

- Pérez, A. (1992). "Enseñanza para la comprensión”. En Sacristán, G. y Pérez, A. Comprender y transformar la enseñanza (pp. 68-107). Madrid: Morata.

- Rodrigo, M. J. Rodríguez P, A. y Marrero, J. (1993).Las Teorías Implícitas: Una Aproximación Al Conocimiento Cotidiano. Madrid: Visor.

- Sánchez, M. D.(2005).Creencias sobre razonamiento moral y Conflictos en adolescentes con Problemas de adaptación escolar [tesis doctoral].Málaga: Universidad de Málaga.

- Uprimny, R. (2001).Orden Democrático y Manejo de Conflictos, Corporación Viva la Ciudadanía. Bogotá: Universidad Pedagógica Nacional.

- Valderrama, C. E. (2001). Nociones del conflicto en actores escolares. Nómadas. 1(15), 76-87.

- Zuleta, E. (1994).Elogio de la dificultad y otros ensayos. Bogotá: Editorial Fundación Estanislao Zuleta. 